\title{
La pobreza, el crecimiento demográfico y el control de la natalidad. \\ Una crítica a la perspectiva ética de Peter Singer sobre la relación entre ricos y pobres
}

\author{
Poverty, demographic growth, and birth control: \\ a critique of Peter Singer's ethical perspective \\ on the relationship between rich and poor
}

Dalia Elena Romero1

\footnotetext{
1 Departamento de Estudios Demográficos, Universidad Católica Andrés Bello, Venezuela.

Urb. Montalbán-La Vega, Apartado 29068, Caracas, Venezuela.
}

Abstract This articleanalyzes the relationship between population growth and ethical principles relating to poverty. The paper is a critical approach to the thesis presented by Peter Singer in his book "Practical Ethics". The first part briefly examines the principal topics of his thesis. The author then analyzes the basis of Singer's theory with respect to the following questions: 1) Is overpopulation the main reason for poverty? Is it possible to establish an association between the poverty phenomenon and population growth? 2) Is Singer's demographic perspective valid? 3) Can problems of resource distribution be ignored when talking about poverty from an ethical perspective? 4) Is it true that birth control policy was successfully implemented in Mexico, Colombia, and Brazil? 5) Does Singer's position on population growth have a negative influence on the "collective imagi nation"? The paper concludes by suggesting some useful arguments for understanding an ethical perspectivetowards poverty.

Key words Poverty; Population Growth; Birth Control

Resumen En esteartícul o se presenta una reflexión sobre la relación entre el crecimi ento demográfico y la ética frente a la pobreza, a partir de una crítica a los argumentos presentados por Pe ter Singer en su libro “Ética Práctica”. En primer lugar, se colocan en pocas palabras los propios argumentos del autor. A continuación, se analizan los "pilares" que sustentan su argumentación, intentando responder a las siguientes cuestiones: 1) ¿Es el "exceso de población" la princi pal causa de la pobreza? ¿podemos encontrar una relación entre pobreza y velocidad del crecimiento de la población? 2) ¿Está vigente la perspectiva demográfica que Singer asume? 3) ¿Se puede ignorar o restarle importancia al problema de la distribución de los recursos y los ingresos cuando estamos tratando el problema de la pobreza desde una perspectiva ética? 4) ¿Quétan cierto es ese argumento del éxito en los casos de México, Colombia y Brasil en cuanto a la implantación de una política de control dela natalidad? 5) ¿Esa postura del autor sobre el crecimiento dela población tiene consecuencias negativas en el "imagi nario colectivo"? Por último, a manera de conclusión, se presentan algunas cuestiones que deben ser consideradas en una ética frentea la pobreza.

Palabras clave Pobreza; Crecimiento Poblacional; Control de la Natalidad 


\section{Introducción}

A pesar de los avances en el tema de la relación sobre crecimiento de la población y pobreza, todavía se sigue argumentando que uno de los principales males de este siglo es el crecimiento desmesurado de la población, por su responsabilidad en la depredación del medio ambiente y en la pauperización de las condiciones de vida. Ese argumento es divulgado por medios de comunicación, por estudios Ilamados “científicos" e incorporado en el saber popular, a tal punto que pasa a constituir casi un axioma.

Este trabajo está centrado en la reflexión y crítica a las ideas sobre crecimiento demográfico, pobreza y ética que trabaja Peter Singer en su libro “Ética Práctica”, específicamente en el capítulo denominado "Ricos y Pobres". Este autor es filósofo, co-director del Institut of Ethics and Public Affairsy director del Centre of Human Bioethics da Universidade De Monash, Melbourne. Tiene importantes investigaciones y aportes en el campo de la bioética, especialmente sobre la "liberación animal" y en cuestiones sociales controvertidas como la igualdad y discriminación de la raza y el sexo, el aborto, la eutanasia. Es uno de los grandes representantes del utilitarismo consecuencialista, denominación con la que el mismo se identifica (Singer, 1994:11-22). En el capítulo antes citado sobre ricos y pobres, Singer desenvuelve su argumentación sobre la ética frente a la pobreza como una cuestión que se traduce principalmente en la obligación de ayudar a los otros y que tiene como condicionante que esos países pobres disminuyan su población.

En este trabajo se trata de reflexionar específicamente sobre algunos conceptos que Singer utiliza en su argumentación en relación al tema de población y el crecimiento demográfico (el autor utiliza el término crecimiento de la población como "crecimiento natural", no hace referencia al otro componente del crecimiento, los movimientos migratorios, o migración neta, Pressat, 1983), ya que esas son algunas de sus principales "herramientas" para construir su argumentación utilitarista consecuencialista sobre la obligación de ayudar a los pobres. La cuestión que me preocupa en ese discurso y argumentación es principalmente ¿cuales son las consecuencias, desde el punto de ética práctica, que provocaría el aceptar como una verdad que existe un "exceso poblacional" el cual a su vez es el principal responsable de la proliferación de la pobreza?.

En el supuesto negado de que el crecimiento de la población fuese el responsable, ¿esa perspectiva utilitarista-consecuencialista, que promulga que los países más ricos nieguen su ayuda a los países pobres que no acepten el uso de métodos anticonceptivos para la disminución de la fecundidad, no está en contradicción con principios importantes de la ética como es el de autonomía? Son preguntas que entran más específicamente en el campo de las concepciones éticas y, por lo tanto, no se discutirán en el presente trabajo; sin embargo, vale señalar que existe una discusión en cuanto a la "neutralidad" de los argumentos sobre "maximización de intereses" relacionada con el enfoque utilitarista-consecuencialista. Una de las perspectiva de análisis que se podría asumir dentro del mismo campo de la ética son las revisiones sobre utilitarismo, y/ o un análisis lingüístico. Como no es esa la finalidad de este trabajo, sólo hago referencia a algunas reflexiones realizadas por Jurandir Freire Costa, las cuales considero pertinentes en esta ocasión (Costa, 1994). Él considera que el utilitarismo pretende derivar convicciones éticas de hechos o realidades empíricas, donde las creencias morales son aceptadas en la medida que atienden a la mayor satisfacción posible de intereses, todos ellos concebidos como hechos preexistentes a interpretaciones. Jurandir retoma las críticas que al respecto realiza Wittgenstein, en las que este último afirma que "reducir las creencias morales a representaciones ideales de hechos empíricos significa, la mayoría de las veces, disimular: a) que lo que se describe como "realidad material", como hecho bruto, nada más es que la "realidad lingüística", sin soporte temporal-espacial, como en la efectiva realidad material de los objetivos físicos; b) que estas "realidades lingüísticas" no son neutras en cuanto a valores, lo cual queda evidente (sic) cuando se observa el papel de los “intereses" en el utilitarismo" (Costa, 1994:32).

Entonces, se pregunta Jurandir, ¿qué quiere decir "maximizar" cuando se dice que una convicción moral deriva de la finalidad de maximizar intereses? “¿cómo se procede a esa medición? ¿cual es el patrón al que se recurre para reconocer que un cierto interés será “maximizado" si tal creencia fuese asumida? ¿qué es lo "máximo" y lo "mínimo" en este caso?" (Costa, 1994: 32). Desde esa perspectiva sobre el enfoque utilitarista-consecuencialista, es importante revisar cuales son los "hechos observables" que sustentan su relación costo-beneficio. 


\section{Argumentación de Peter Singer}

Su argumentación se desarrolla de la siguiente manera:

Primera Premisa: Si pudiésemos impedir que algo ruin suceda sin tener que sacrificar algo de importancia comparable, debemos impedir que suceda.

Segunda Premisa: La pobreza absoluta es algo ruin.

Tercera Premisa: Existe una parcela de pobreza absoluta que podemos impedir sin que sea preciso sacrificar nada de importancia moral comparable.

Conclusión: Debemos impedir la existencia de una parcela de la pobreza absoluta.

En primer lugar, él reflexiona sobre la crueldad de la pobreza absoluta. Algunos de los indicadores que señala son: 400 millones de personas no tienen las calorías, las vitaminas y los minerales necesarios para mantener sus cuerpos y sus mentes en condiciones saludables; 14 millones de niños menores de cinco años mueren anualmente por mala alimentación e infecciones y 180 millones viven en condiciones de extrema desnutrición. Sin embargo, a pesar de que reconoce que el problema no es que el mundo no sea capaz de producir lo suficiente para alimentar su población (por ejemplo, Estados Unidos consume en media 5 veces más granos que los países pobres), sino que, en los países ricos, se alimentan los animales con la mayor parte de los granos y luego lo transforman en productos lácteos (pág. 231), no profundiza en el problema de la distribución de recursos, alimentos, bienes, entre otros, como causa de la pobreza. Su planteamiento sobre la desigual distribución es simplista, pues lo reduce a la cuestión de los granos “mal utilizados" en los países ricos.

Llama la atención que el autor no realiza juicios éticos sobre la responsabilidad que tienen los ricos absolutos sobre la pobreza, porque considera que, dentro de su perspectiva consecuencialista, es mejor evitar entrar en censuras que cohiban la "obligación de ayudar", es decir, al censurar a los ricos sería más difícil persuadirlos para que ayuden con dinero a los pobres: “...no pretendo estar emitiendo jui cios éticos sobre la riqueza absoluta, apenas Ilamar la atención para el hecho de ella existir..." (Singer, 1994: 233).

Su tercera premisa se refiere directamente al compromiso ético que los ricos deben asumir ante la pobreza absoluta, algo que pueden hacer sin sacrificar alguna cosa de importancia moral comparable. Peter Singer considera que ese último principio deben aceptarlo todos, tanto los consecuencialistas como los no-consequencialistas.

Desde su postura utilitarista de "mejores consecuencias", en la cual, una vez analizadas todas las alternativas, se deben favorecer los intereses de los que son afectados (Singer, 1994:22), el autor considera que, en lugar de "configurar una ética para santos y héroes", que exija algo exagerado que nadie cumpla, es preferible un nuevo abordaje donde se logren compromisos concretos aunque limitados de parte de los ricos. Por ello, no desea entrar en la discusión sobre el contraste entre la riqueza y la pobreza, sino examinar su ética ante la pobreza: "compromiso de ayudar", en el contexto actual del mundo (Singer, 1994:240).

“Tal vez la más seria objeción al argumento de que tenemos obligación de ayudar sea a que, como la mayor causa dela pobreza absoluta es el exceso de población, ayudar a los que hoy viven en situación de pobreza apenas aseguraría que más personas naciesen para vivir en la pobreza del futuro" (Singer, 1994:247).

Más, ese argumento para "obligar éticamente" a que los ricos absolutos ayuden con dinero a los pobres absolutos se tropieza con algunos hechos que ponen en duda la objetividad de su afirmación.

Él encuentra la solución a esa disyuntiva en la teoría sobre transición demográfica, la cual la reduce a la afirmación que, cuando aumenta el patrón de vida, disminuye la población. En ese sentido, afirma, no se puede permitir una política de "triaje", cuando existe la posibilidad de que el crecimiento de la población pueda ser controlado sin recursos crueles y "selectivos".

Vale señalar que existe una amplia crítica sobre la interpretación de las Ilamadas "etapas demográficas" y sus explicaciones. Especialmente en América Latina se dio un fuerte debate político-ideológico en los años 70 que se puede encontrar en Patarra, 1973; Mertens, 1982; Szreter, 1993. Aunque se reconozca que la línea de investigación sobre transición demográfica es sumamente importante, existe una serie de limitaciones que es preciso tener en cuenta. Entre ellas se encuentra que muchos esperan que las etapas estén acompañadas de procesos socio-económicos similares a los de los países que dieron origen a la teoría; por otra parte, la teoría no puede generar hipótesis claras en cuanto a las causas específicas de los cambios de la fecundidad.

Afirma Singer que una manera de asegurar éxito a esa ayuda, y por lo tanto "compensar" los sacrificios que hacen los ricos al ayudar a los pobres, es condicionando esas "ayudas" al control del crecimiento de la población de esos 
países pobres, especialmente a través del incentivo de uso de técnicas de control de la natalidad. Esas técnicas, según el autor, han presentado una excelente relación costo-beneficio. Por lo tanto, la ética de triaje se tornaría inaceptable, es decir, aunque los que promulgan el control de la población a través de la hambruna a gran escala y de las enfermedades tengan razón, según Peter Singer, en cuanto a que ayudar a los pobres traería más problemas que beneficios por el crecimiento natural de la población, sus argumentos dejarían de tener fuerza si se reconoce que existen maneras efectivas de controlar el tamaño de la población, de evitar que más personas naciesen para vivir en la pobreza, en el futuro (Singer, 1994:252).

El autor plantea entonces un dilema: cómo decir que, desde una postura ética, se debe ayudar a los pobres si tienen razón los defensores de la "selectividad" o "triaje" en cuanto a que, si se ayuda a los pobres, sólo se va a ayudar a reproducir a la pobreza, si, a largo plazo, cuando la capacidad de ayuda expire, el sufrimiento será mayor de lo que sería si la ayuda se detuviese ahora. ¿Cómo ser éticos sin pensar en las consecuencias a largo plazo de nuestras acciones? El autor se preocupa con esa solución que la propuesta de triaje propone: dejar que el crecimiento natural de la población, especialmente con el aumento del índice de mortalidad de los más débiles, de los que mueren por infección, desnutrición y otras enfermedades que suelen acompañar a los pobres, acabe con la pobreza.

Aunque señala que, entre esos recursos alternativos para ayudar a los países a elevar el patrón de vida de los miembros más pobres de su población, está el de incentivar a sus gobiernos a realizar una reforma agraria, elevar el nivel educativo e impedir que el único papel de la mujer sea el de la procreación; sólo se detiene en las consecuencias del último propuesto: ayudar a otros países a tornar más disponibles las técnicas de control de la fecundidad y de la esterilización. Coloca como ejemplo exitoso de ese último recurso los casos de México, Colombia y Brasil.

“...Tratase de hecho de una imposición, más ¿será ella injustificable?. Si el argumento a favor dela obligación deayudar es bien fundado, tenemos la obligación de reducir la pobreza absoluta; no tenemos, sin embargo, la menor obligación de hacer sacrificios que, hasta dondesabemos, no ofrecen perspectiva alguna de reducir la pobreza a largo plazo. Por tanto, no tenemos ninguna obligación de ayudar a países cuyos gobiernostienen políticas capaces de tornar a nuestra ayuda inoperante..." (Singer, 1994:253).
Argumentos "empíricos" que sustentan su argumentación: una revisión

Debido a que los utilitaristas-consecuencialistas, como Peter Singer, se basan en hechos, es crucial revisarlos para reconocer si esa relación entre costo-beneficio que proponen puede tener el resultado esperado $y$, por lo tanto, tener rendimientos positivos. Sin duda, la argumentación de Peter Singer presenta un orden lógico estricto. Sin embargo, esos hechos que toma como "pilares" son los mismos que ponen en duda la solidez y la irrefutabilidad de su propuesta.

¿Es realmente el "exceso de población" la principal causa de la pobreza? ¿se puede llegar a una relación entre pobreza y velocidad de crecimiento de la población?

Ese concepto de "excedente de población" parte del supuesto de que existe una relación negativa entre el crecimiento económico y el crecimiento demográfico; en otras palabras, consideran a la población como un freno para el desarrollo (Coale \& Hoover, 1958; Simon, 1980; Watkins \& Walle, 1983). Durante la década de los sesenta esa relación entre población y desarrollo toma auge a raíz de las acciones del control natal liderado por los Estados Unidos. La idea más difundida es que los problemas de pobreza de un país se resuelven con la disminución del crecimiento demográfico. El embate ideológico que surge de ese enfoque se resume en la famosa frase del entonces presidente de los Estados Unidos que decía que "cinco dólares en control de la natalidad rinden más que cien en desarrollo" (Fucaraccio, 1994: 73). Fucaraccio realiza un excelente trabajo en el cual examina los autores clási cos y los distintos enfoques que han dado al tema y su relación con el entorno económico-social y político donde producían su conocimiento. El señala que, en los últimos veinte años, en el continuo desarrollo de la polémica, se han enfatizado aspectos parciales de ese pensamiento, olvidándose la realidad concreta para la que se formuló (Fucaraccio, 1994:73). Es allí que interesa detenerse el presente artículo, ya que la postura de Peter Singer está impregnada de esa polémica.

La relación entre la población y el desarrollo se ha enfocado tradicionalmente como "determinantes y consecuencias" (un ejemplo de este enfoque, que tuvo amplia repercusión, fue el trabajo de Naciones Unidas, 1978). Los determinantes se consideran los factores que "explican" el comportamiento de la fecundidad, mortalidad y migración; y, en las consecuen- 
cias, se estudia cómo afectan a las variables económicas y sociales el tamaño y el crecimiento demográfico, la estructura por edad, entre otros. Algunas de esas preguntas tienen que ver en con la influencia del crecimiento de la población en el ahorro, la inversión, la ocupación. Sin embargo, como señala Fucaraccio, hasta hoy no se han logrado conclusiones firmes (Fucaraccio, 1994:74) y por el contrario, se han real izado sólidas críticas a ese argumento sobre el efecto negativo del crecimiento de la población sobre el ahorro y la inversión (Fucaraccio, 1970; Singer, 1976; Simon, 1980). Encuentra que la evolución del pensamiento en la teoría económica en cuanto al rol de la población puede verse como olas recurrentes. En un primer momento, la población aparece como beneficiosa para el desarrollo económico: es el mercantilismo y Smith; en un segundo momento, la población aparece como un obstáculo al desarrollo: es Malthus; en un tercer momento, con Marx, la dinámica demográfica aparece como un mero reflejo del sistema capitalista; un cuarto momento, en el cual se ignora la contribución de Marx y se inicia el examen de "determinantes y consecuencias"; un quinto momento, por el cual el pensamiento retorna a Malthus, pero bajo la terminología económica y, por último, un retorno al tratamiento sistémico que iniciara Smith, pero enriquecido y modificado con el ementos sobre la realidad del subdesarrollo (Fucaraccio, 1994: 78).

El concepto de "excedente de población" u “óptimo de población", utilizado por Peter Singer para sustentar su postura sobre la reproducción de la pobreza y la ética frente a esta, es un concepto totalmente superado. Prácticamente terminó en los años cincuenta y estuvo vinculado con la tesis de Malthus. El problema que plantea es la determinación de la tasa de crecimiento de la población compatible con la tasa de crecimiento del producto. La conclusión se torna vacía principalmente por la introducción del cambio tecnológico (Fucaraccio, 1994:82; otros autores sobre este tema: Peacock, 1952; Tapinos, 1994). Los problemas de los que se derivaban ese enfoque de los años cincuenta provienen de la preocupación de los centros de poder de los Estados Unidos en cuanto a que, por las tensiones que generaba la pobreza, se implantase el socialismo en eses países. Como señala Fucaraccio (1994:84), sus argumentos (con el principal trabajo de Coale y Hoover) ni siquiera sus mismos propulsores hoy lo defienden. Un experimento reciente realizado en Filipinas (Ilamado BACHUE) es un modelo para simular la planificación económica, social, y demográfica. La principal conclu- sión que arroja en relación a población y crecimiento económico es que la caída rápida de la fecundidad no es una panacea para resolver problemas económicos y, más, tiene poco impacto en los resultados económicos, laborales, entre otros (Rodgers et al., 1977).

¿Está vigente esa perspectiva demográfica que Singer asume? ¿existe consenso entre las diferentes perspectivas?

Dentro del desarrollo del pensamiento demográfico, se puede decir que posturas como las que asume Singer están cargadas de pre-conceptos que se identifican con una perspectiva catastrofista. De esas perspectivas, hoy en día, subsisten tres principales corrientes: están el ecologista, el demográfico y el del desajuste de la demanda y la oferta de capital. El primero de ellos es el más antiguo. Sustentan que los recursos naturales son limitados, y necesariamente llegará el momento en que la población creciente se enfrente a la restricción de los recursos naturales. El segundo, el demográfico, subraya la importancia de las variaciones de la relación de dependencia resultante del control de la fecundidad. El último habla del desajuste entre el crecimiento poblacional, es decir, la alta fecundidad, la cual disminuye la oferta de capital ya que supone que existe una relación inversa entre la capacidad de ahorro y la fecundidad y, por otro, el incremento de población exige inversión de capital, Ilamada, a veces, inversión demográfica. Como se ha mencionado anteriormente, esos argumentos, según diversos analistas, carecen de fundamentación en la actualidad (Tapinos, 1994: 65).

Existe un nuevo enfoque, el cual tenemos que tener muy en cuenta, por sus aportes en el estudio de la problemática de la población, que, de forma resumida, puede decirse que parte de una nueva visión de la articulación población-acumulación de capital-progreso técnico. Ese enfoque demuestra que el crecimiento demográfico, en si mismo, no tiene por efecto inhibir el crecimiento económico. En particular, demuestra que la relación negativa entre la fecundidad y el ahorro de los hogares carece totalmente de verificación empírica robusta. De conformidad con esa perspectiva, se ha demostrado más recientemente el efecto positivo del crecimiento demográfico sobre la innovación y sobre la difusión de la innovación (Tapinos, 1994:67). En esa perpectiva, el crecimiento económico no se toma sólo como un crecimiento de los actores de producción y un crecimiento de la productividad, sino especialmente como un proceso de transformación es- 
tructural que implica cambio institucional. Por ejemplo, en el análisis de la crisis de alimentos, se muestra que el problema no está en la relación con la insuficiencia de la oferta, sino más bien con la organización del sistema productivo y distributivo. Es decir, los efectos económicos del crecimiento demográfico toman cuerpo realmente en las variables institucionales (Tapinos, 1994:67-68).

La perspectiva ecologista es la más cercana a la de Peter Singer (se autodefine dentro de la corriente de "animal liberation" y ecología profunda). En general, ellos promulgan que el crecimiento demográfico atenta contra el medio ambiente. No se desarrollará en este trabajo esa discusión, por dos razones. En primer lugar, porque el objetivo es la discusión sobre crecimiento demográfico y pobreza y, en segundo lugar, porque el mismo Peter Singer, en el capítulo sobre medio ambiente (Singer, 1994:279304), reconoce que los principales problemas en la relación hombre-naturaleza y en el respeto por la vida están en los países desarrollados, por sus patrones de consumo.

Mucho se ha escrito sobre la falacia de esa relación directa entre crecimiento demográfico y medio ambiente. Sólo mencionaré lo que muestran algunos indicadores publicados por un suplemento de las Naciones Unidas (Population Reports, 1994), que dan idea de cómo es mayor la contaminación y el deterioro al medio ambiente que están ocasionando países desarrollados con bajas tasas de natalidad. Mientras en América Latina el consumo promedio de energía comercial en 1989 es de 24 gigajoules, en Europa Occidental es 6,4 veces mayor y en Estados Unidos ese promedio es 12 veces mayor que en América Latina (153 y 295 gigajoules); así mismo, la emisión de dióxido de carbono per-cápita en América Latina es inferior que en Europa Occidental 5,4 veces y menor que en Estados Unidos 12,3 veces (1,6; 8,6 y 19,7 toneladas métricas de dióxido de carbono, respectivamente). Vale la pena mencionar que, en las reuniones preparatorias a la Conferencia Mundial de Población de 1994, que se dieron por tres años consecutivos en cada país y entre las regiones, una de las cuestiones que los países no desarrollados reivindicaron de la primera versión para la discusión propuesta por Naciones Unidas fue en relación a la población y el medio ambiente. Se consiguió explicitar que el crecimiento de la población de los países no desarrollados no es uno de los principales responsables del deterioro del medio ambiente.
¿Se puede ignorar o restarle importancia al problema de la distribución de los recursos y de los ingresos, cuando estamos tratando el problema de la pobreza?

En lo que se refiere a la distribución del ingreso y su relación con el crecimiento de la población, vale la pena mencionar que una reducción del crecimiento demográfico no tiene efecto sobre la distribución del ingreso, es decir, no genera beneficios a largo plazo para los pobres, aunque algunas ganancias se obtengan en el corto plazo y, marginalmente, puedan reducir la desigualdad (McNicoll, 1984). Eso porque, una vez configurada una distribución del ingreso estable en el tiempo, las inversiones se orientan a satisfacer necesidades en los grupos de mayores ingresos que, a su vez, reproducen las condiciones de distribución. Por ello, encontramos ejemplos como el de Brasil, el cual, a pesar de haberse transformado en la octava potencia industrial del mundo (Kliksberg, 1993), y paralelamente, disminuido su fecundidad con una velocidad pocas veces experimentada en otros países (Berquó, 1990; 1994a) tuvo su situación social deteriorada drásticamente, colocándose entre los países con mayor desigualdad en el mundo. Según cifras de las Naciones Unidas, en 1981, el 50\% de la población tenía el $13,4 \%$ del ingreso nacional; del otro lado, el $1 \%$ tenía casi la misma participación, el 13\%. Esa estructura absolutamente desigual se agudizó en 1989: el 50\% de la población redujo su participación al 10,4\% del ingreso nacional y el 1\% más rico aumentó al 17,3\% (Naciones Unidas, 1990).

“La historicidad y el timing de la transi ción demográfica en América Latina también constituyen una base empírica suficiente para contraponer a los argumentos neomalthusianos de asociación entre crecimiento poblacional y pobreza. La verdad, esasúltimas décadas de declinio dela fecundidad no fueron acompañadas deuna mejoría delas condiciones devida de los grupos social es envueltos; al contrario, coincidieron con el final dela perspectiva desarrollista, con el Estado deficitario y en la profundización de las desigualdades sociales" (Patarra, 1994:34).

Entonces, si se quiere plantear una ética frente a la pobreza, como lo intenta Peter Singer, hay que tener muy presente que el crecimiento demográfico no ha tenido incidencia en la creciente subutilización de la fuerza de trabajo urbana, así como tampoco en la creciente pobreza, sino es la peculiar manera de la dinámica capitalista que, con su propia dinámica, genera esos problemas (Fucaraccio, 
1994:97), los cuales hoy en día los podemos ver más claramente cuando no es más la fuerza de trabajo, sino el salto tecnológico y la internacionalización del capital los que definen el nivel de desarrollo capitalista de un país.

¿Fueron realmente exitosos los casos de México, Colombia y Brasil en cuanto a la implantación de una política de control de la natalidad?

Singer afirma que existen ejemplos exitosos en los cuales el uso de las técnicas de control de la natalidad y la esterilización fueron efectivos en la aceleración de la transición demográfica, tal como México, Colombia y Brasil (Singer, 1994: 252).

En primer lugar, es importante señalar que ese tipo de afirmaciones, tan generalizado en el medio académico, no sólo empobrece la caracterización de la transición demográfica sino, además, llega hasta a distorcionar la complicada red de causas y consecuencias de la transición demográfica que se dan en el ámbito social, cultural, familiar, de género, de status de la mujer, entre otros. En otras palabras, se debe tener claro que el uso de métodos anticonceptivos y de la esterilización es uno entre diversos factores que incide en el declinio de la fecundidad; que, por otra parte, es uno de los mecanismos y no una causa directa de la enorme disminución de la fecundidad (Fonseca \& dos Santos, 1993), por lo menos en los países mencionados como ejemplo por Peter Singer; que, si bien es cierto que el uso de métodos anticonceptivos aceleró la transición demográfica en muchos de los países pobres, no hay que despreciar que, en muchos casos, esa práctica estuvo desligada de acciones relacionadas con salud de la mujer; que se realizó con coerción y hasta con coacción y, por lo tanto, se asumieron una serie de costos en el plano de los derechos humanos (Berquó, 1990); que se provocó una serie de efectos directos e indirectos en lo que dice respecto a relaciones de género (Fonseca \& dos Santos, 1993), asi como distorciones en la composición por edad de la población.

En ese sentido, más que el uso de anticonceptivos y de la esterilización femenina como medios para disminuir la fecundidad en México, Brasil y Colombia (países mencionados por Peter Singer), se deben considerar las series de mudanzas estructurales socio-económicas tal como los procesos de modernización, la participación femenina en el ámbito laboral, la escolarización de la mujer, las condiciones culturales, el valor de los hijos como causas de esa disminución (Davis \& Blake, 1955). Más recien- temente se ha demostrado que la división entre los espacios domésticos y públicos entre mujeres y hombres, respectivamente, la cuestión de poder embutida en el concepto de género y el status y el proceso de decisión de la mujer (Mason, 1988) constituyen un enfoque con mucho potencial para explicar mejor tanto las causas de la transición demográfica como sus consecuencias. Quizá uno de los casos que ha sido más estudiado bajo ese último enfoque sea el de Brasil.

En Brasil la disminución de la fecundidad comienza a ser observada más claramente a partir de la década de los setenta, pero es desde mediados de la década anterior que se define la tendencia hacia el decline, con la masificación de los métodos anticonceptivos modernos y el método irreversible de esterilización (Carvalho \& Wong, 1990). Sin embargo, para entender el proceso es importante señalar que en esas décadas se dieron cambios radicales en el nivel educativo, en la participación laboral, en las condiciones económicas y culturales y en las estrategias de sobrevivencia de las mujeres brasileñas y de sus hogares, que no necesariamente implicaron una mejora de las condiciones de vida de la población femenina. Por otro lado, dada la enorme desigualdad económica, de recursos y de desarrollo que se encuentra en Brasil, no se pueden generalizar las causas de la disminución de la fecundidad. La clase de mujeres más empobrecidas que se incorporó a la fuerza de trabajo, más por presiones económicas que por mudanzas en sus expectativas de vida, con condiciones de salud hasta empeoradas, ha sufrido el violento impacto de las mudanzas en la concepción sobre relación hombre-mujer, mujer-madre cambios estes que no fueran acompañados por políticas de salud reproductiva y mudanzas institucionales que permitan aliviar la carga que ha tenido la transición demográfica sobre la mujer. Un ejemplo de ello es el alto número de abortos practicados diariamente sin condiciones mínimas de salud y de apoyo psicológico. Es decir, por un lado, se le impone a las mujeres un modelo de mujer independiente, trabajadora y responsable del hogar y de su ámbito de trabajo y, por otra parte, no se les brinda apoyo institucional para realizar tales metas ni para ejercer la sexualidad y la fecundidad de una manera libre y conciente.

En relación a la salud reproductiva, en muchos de los países, como el Brasil, se presenta un panorama desalentador. A lo largo de la historia el énfasis de atendimiento fue dado a la díada niño-madre; en otras palabras, a la asistencia materno-infantil (Fonseca $\&$ dos Santos, 
1993), lo que implica el desplazo de la salud de la mujer a su sola condición de reproductora. En ese sentido, la ausencia de un programa de salud reproductiva centrado en la mujer, en el cual la reproducción y su planeamiento fuese uno de los componentes y no el único eje, hizo que las mujeres se restringieran apenas a los métodos hormonales y al método irreversible de esterilización.

En relación a ese último aspecto Berquó, haciendo referencia a diversas investigaciones, señala que esa restricción es producto de que las acciones estatales en relación a la natalidad tienen más que ver con un control stricto sensu, por la urgencia que tienen en cumplir con los compromisos asumidos con diversos organismos internacionales para reducir el ritmo de la fecundidad. De hecho, en la década de los años setenta el gobierno estimuló la práctica de acciones privadas para el control de la natalidad con recursos financieros provenientes del exterior, sin imponerles medidas de control y vigilancia (Berquó, 1990:87).

La autora señala que esa falta de programas públicos sobre salud sexual que ofrezcan libremente toda una gama de métodos anticonceptivos y las dificultades para el acceso a los anticonceptivos acaban por colocar a las mujeres frente a una encrucijada: o la esterilización, o el aborto, o un hijo no deseado. La realización de la esterilización, en muchos casos, se da fuera de las condiciones exigidas, gracias a la complicidad que se establece entre mujeres y médicos. Su aplicación de forma masiva es una de las peores consecuencias de esa política antinatalista, debido a sus graves consecuencias en cuanto a los efectos psicológicos, de pareja, que ocasiona la utilización de métodos irreversibles, sobre todo entre las mujeres jóvenes. En Brasil esos niveles de esterilización en mujeres jóvenes (Berquó, 1994a) Ilegaron a números que no encuentran paralelo en ninguno de los países desarrollados.

Uno de los grandes avances de la última Conferencia de Población realizada en el Cairo, en 1994, fue en cuanto a la discusión y hasta pronunciamiento en torno a la equidad entre los sexos y en cuanto a los derechos reproductivos y la salud sexual y reproductiva. Entre los objetivos se planteó propiciar y apoyar decisiones responsables y voluntarias sobre la procreación y los métodos de regulación de la fecundidad y disponer de la información, la educación y los medios para ello, asi como atender a las nuevas necesidades sobre salud reproductiva durante todo el ciclo biológico, y hacerlo de un modo que respete a las diversas circunstancias de las comunidades locales. Es intere- sante observar que, entre las medidas más discutidas en dicha conferencia, está la que menciona el deber de la comunidad internacional de brindar apoyo a los programas de los países en desarrollo, cuando se le solicite, y de no comprometerlos sobre capacitación, asistencia técnica, suministro de anticonceptivos a corto plazo. Al mismo tiempo, afirman que, en esos países, se debería dar más importancia a los servicios de atención de la salud reproductiva y sexual, incluyendo una amplia gama de medios anticonceptivos, y se debería abordar la práctica actual de recurrir al aborto para regular a la fecundidad, satisfaciendo urgentemente la necesidad de las mujeres de esos países de contar con mejor información y más opciones. Gran parte de los países de América Latina llevaron y defendieron la posición en cuanto a la total soberanía que los Estados nacionales deben tener en la definición de sus políticas; la necesidad de distanciamiento de posiciones neomalthusianas; la planificación del número de hijos sin coerción, con información, con libertad y como derecho fundamental; así como la necesidad de integrar la planificación familiar a los servicios públicos de salud.

El caso mexicano también ha sido motivo de amplias discusiones. Es uno de los pocos países que se comprometió con una tasa específica de reducción del crecimiento de la población, por lo cual realizó grandes campañas, programas y acciones reducidos al uso de anticonceptivos y de la esterilización que, como en el caso de Brasil, estuvieron, en muchos casos, lejos del respeto a los derechos humanos y de la realidad social y cultural de la familia mexicana. Además, a pesar de la intensa disminución de natalidad registrada, se han desatado las más fuertes crisis económicas, sociales y políticas de este siglo. Como señala un importante demógrafo mexicano:

“Las reacciones anti estatal es surgen en primer lugar porque precisamente no se cumplen los derechos humanos, ya que la libertad está condicionada. Por un lado, está condicionada por la propia situación social: los pobres disfrutan de menos "libertad para decidir" quelos ricos y los del campo no disfrutan de ninguna opción. No se contemplan con un mínimo de equilibrio los aspectos económicos, social es y culturales. Las condiciones de vida continúan siendo tan o más desiguales que hace veinte años. A pesar del crecimiento económico menor, no acontece una mejora importante" (Benítez, 1987:25).

Uno de los avances importantes en la última Conferencia de Población y Desarrollo realizada en el Cairo es que ningún país presentó 
metas relacionadas con reducciones explícitas del crecimiento poblacional (Berquó, 1994b).

Entonces, considero que ninguna ética sobre la pobreza puede desconsiderar los hechos mencionados anteriormente y, menos aún, como la propuesta por Singer (1994) la cual, bajo su óptica de ética práctica, considera la disminución de la fecundidad en los países pobres, a través de la esterilización y métodos anticonceptivos.

¿Esa postura sobre el crecimiento de población tiene consecuencias negativas en el "imaginario colectivo"?

También hay que considerar que, cuando se apoya la idea de que el crecimiento de la población es el problema, como lo realiza Peter Singer (1994), se están propiciando consecuencias negativas en el imaginario colectivo. Denis Duclon, sociólogo, director de investigación del Centre National de la Recherche Scientifique (CNRS), en su artículo “Quand la culture dela haine s'approprieles technologies de mort", publicado en Le Monde Diplomatique de agosto de 1996, realiza un interesante análisis sobre los recientes atentados de masas ocurridos en el mundo. El autor Ilama la atención, en primer lugar, para la facilidad con la que grupos extremistas se apropian de tecnologías letales y, en segundo lugar, para el vertiginoso crecimiento de una "cultura silenciosa" de la violencia que se está extendiendo en el mundo. Esos grupos que promueven la cultura de la violencia tienen como base ideológica las previsiones apocalípticas del mundo en caso de que no se controlen a las diferentes minorías (como raciales y religiosas) a través de acciones extremistas. Considera que el trabajo del imaginario en la cultura no es inofensivo:

"nos acostumbra a perspecti vas de pesadillas demográficas como si se tratase de lo real, con un consenso tácito que parece admitir la inevitabilidad deuna pandemia exterminando a una parte dela población humana, de preferencia sub-desarrollada" (Duclon, 1994:22).

Duclon critica que, sin ninguna base científica, muchos grupos banalizan la idea de que la explosión demográfica en África, América Latina e India acompaña fatal mente al recrudecimiento de virus como el HIV, y prepara el terreno para la aceptación de la aparición "natural" de una pandemia exterminadora. Esa visión apocalíptica, según él, estimula la "cultura de la violencia" y crea una ideología de aceptación de las acciones de grupos extremistas. Por ejemplo, los atentados ocurridos en 1995 en Oklahoma, en el metro de Tokio y en el metro de París tienen en común que sus promotores y ejecutores afirman que se debe eliminar el "excedente de población" responsable de los males sociales, el cual se refiere, generalmente, a negros, migrantes, aidéticos y/ o pobres.

Escenarios como el que describe Denis Duclon podemos encontrarlos en ciudades como Rio de Janeiro, donde el imaginario colectivo en relación a la pobreza y el crecimiento demográfico han hecho que gran parte de población justifique acciones tan crueles como la matanza de "niños de la calle" por grupos exterminadores. Considero que el argumento que sigue esa racionalidad tiene que ver con que esos niños pobres y abandonados no son "humanos", son productos de un error de la sociedad al no impedir su nacimiento. Es común escuchar en conversaciones, desde analfabetos hasta de intelectuales, que el matarlos hoy evita consecuencias peores para el mañana, tanto para esos niños de la calle potencialmente adultos, como para la sociedad en general. Encontramos también muchas personas que critican de esas matanzas los medios crueles, pero no sus fines. Así, de una forma u otra, se desvirtúan las normas que deberían regir la atención de males tan importantes en la sociedad como la miseria y el abandono infantil. Considero que ese tipo de opiniones refleja lo que Denis Duclon denuncia, la aceptación de la ideología de la eliminación de los grupos considerados inferiores, ideología que acompaña generalmente a esas propuestas "catastrofistas" sobre población.

\section{A modo de conclusión}

Reiterando, es importante que se entienda que el problema de la pobreza está situado en un plano diferente al crecimiento demográfico. En un amplio trabajo presentado por el Programa de las Naciones Unidas para el Desarrollo, “Desarrollo Humano" (Naciones Unidas, 1990), se aporta un interesante análisis del problema de la pobreza. En él se destaca que es la ausencia de compromiso político, y no la falta de recursos financieros, la causa de la miseria. Países más pobres pero con mejor distribución económica logran resultados sorprendentes. Las tasas de esperanza de vida, alfabetización, entre otras, son muy superiores en Costa Rica que en Kuwait, a pesar de que su ingreso per cápita es cuatro veces menor (Kliksberg, 1993). Por lo tanto, considero que, si el ingreso per-cápita no está diciendo de la pobreza real de un pueblo, por qué seguirlo utilizando como indicador de desarrollo? Más aún, ¿por qué considerar que el denominador de su expresión, la po- 
blación, es el obstáculo principal para el desarrollo? Aunque sea cierto, en términos aritméticos, que la menor cantidad de población dará un resultado mayor del ingreso per-capita, en términos de calidad de vida, distribución de la riqueza, derechos humanos, no encontramos relación entre ingreso per-cápita y condiciones de vida de la población.

Así, si el crecimiento demográfico no tiene efecto sobre la distribución del ingreso, ¿es posible aceptar que una ética frente a la pobreza, como la de Singer, pueda estar sobre tales argumentos, pueda proponer que deba existir una "reciprocidad" entre las partes: que los ricos deban "ayudar a los pobres" en troca de una reducción de población? Si hemos visto que ese menor número de población no ha beneficiado a los pobres, ¿quien se está beneficiando entonces? ¿por qué tanto interés de parte de los países ricos en ver disminuido ese "excedente de población"? Así, si los países ricos son los que están deteriorando más el medio ambiente y los que acabaron antes con sus bosques, zonas verdes y recursos naturales en general, ¿por qué utilizar un argumento neomalthusiano para justificar esa Ilamada "reciprocidad entre partes"?

Por otra parte, ¿cómo los países ricos pueden aplicar el mismo razonamiento ético para su propia población, cuando han visto aumentar considerablemente el número de miserables en el seno de su propia sociedad?, ¿se justificaría identificar a los extranjeros como los responsables y eliminarles toda ayuda y servicios sociales que merecen como ciudadanos (como educación y salud), hasta que controlen el "exceso" de su población?; zapoyaríamos la propuesta del congreso conservador de los Estados Unidos sobre la negación de ayuda del Estado a las madres solteras bajo el supuesto de que ellas están, en la mayoría de los casos, reproduciendo la pobreza?

Además, el respeto a la autonomía, uno de los principios cruciales de la ética, según el propio Peter Singer (Singer, 1994:205), dice que debemos permitir que los agentes racionales vivan sus vidas de acuerdo a sus propias decisiones autónomas, libres de coerción o interferencia y que sólo acepta raras excepciones justificadas en razón de justicia, beneficencia y evitar males mayores. Entonces, ¿podemos decir que, en el caso de la ética frente a la pobreza propuesta por Singer, se justifica la violación del principio de autonomía, al condicionar los países y familias pobres a recibir ayuda sólo en el caso que disminuyan su fecundidad y tengan menos hijos?, ¿se trata de normas que orientan la interacción de los ricos con los pobres o sim- plemente la justificación ideológica de la donación y caridad a aquellos países y familias que acepten sus condiciones?

En la Conferencia Mundial sobre Social Management de Copenhague (julio 1991), existió un consenso en cuanto a que el tema de la pobreza es un tema de derechos humanos básicos (Kliksberg, 1993:12). En la última reunión del Club de Roma, se señaló que la pobreza es el gran problema que tiene por delante la humanidad y se resaltó la necesidad de superar la actual:

"insolidaridad delos países ricos para con los pobres... y combati r las di ferentes muestras de egoísmo que se manifiestan tanto en el terre no material como en el espiritual... con el fin de inculcar la solidaridad mundial como ética suprema de supervivencia" (Kliksberg, 1993:12).

Vale la pena también reflexionar en torno a la afirmación del director para América Latina y el Caribe del Programa de las Naciones Unidas para el Desarrollo, Eduardo Zumbado, Ia cual señala la importancia de la solidaridad entre los países y la necesidad de parar con la tendencia actual a imponer barreras económicas a los países pobres.

“El problema dela pobreza tieneimplicaciones éticas, económi cas y políticas de primer orden. Atenta contra los derechos humanos mantener a sectores ampli os de la población en situación de desempleo, desnutrición y marginalidad...

¿Cómo actuar frente a esta situación? Es ne cesario promover un doble frente de consenso internacionales y nacionales. En el plano mundial, es imperiosa la necesi dad de un consenso que abra al Sur los mercados del Norte. Está sucediendo lo contrario, de 20 a 24 países industrializados han subi do sus barreras aduanales consi derablementecon respecto a las quetenían hace 10 años. Son imprescindibles asimismo nuevos tratos que reduzcan el peso de la deuda externa y aumenten las inversiones en el Sur." (Zumbado, 1994: XV).

Para finalizar, vale la pena mencionar que este trabajo no intentó proponer una ética frente a la pobreza sino evaluar, de al guna forma, las bases y conceptos demográficos que "sustentan" la propuesta de Singer en relación al tema. No se trata de criticar su obra, ni desconsiderar los grandes aportes que este autor ha dado al desarrollo de una ética práctica. Sólo llamar la atención sobre algunos pre-conceptos que, si no se los consideran con la debida cautela, pueden tener peores consecuencias en la sociedad y pueden esconder una carga ideológica frente a la pobreza que está fuera de la realidad en la que vive esa población. 


\section{Referencias}

BENITEZ, C., 1987. Y ahora, ¿que hacemos?. Revista Brasileira de Estudos de População, 4:21-31.

BERQUÓ, E., 1990. Uma nova onda para atropelar os direitos reprodutivos. Revista Brasileira de Estudos de População, 7:87-94.

BERQUÓ, E., 1994a. Esterilização e raça em São Paulo. Revista Brasileira de Estudos de População, 11:19-26.

BERQUÓ, E., 1994b. Cairo: o sucesso da arte do diálogo. Revista Brasileira de Estudos de População, 11:109-111.

CARVALHO, J. \&WONG, L. 1990. La transición de la fecundidad en el Brasil: causas y consecuencias. Seminar on Fertility Transition in Latin America. Buenos Aires: International Union for the Scientific Study of Population (IUSSP).

COALE, A. \& HOOVER, E. 1958. Population Growth and Economic Development In Low Income Countries. Princeton: University Press.

COSTA, J., 1994. A ética e o espel ho da cultura. Rio de Janeiro: Rocco.

DAVIS K., \& BLAKE, J. 1955. Patterns favoring high fertility in underdeveloped areas. Eugenics Quarterly, 2:33-39.

DUCLON, D., 1994. Idéologies Folles et Attentats de Masses. Quand la culture de la haine s'approprie les technologies de mort. In: Le Monde Diplomatique. (Ramonet, org.), pp. 22-23. Publicación mensual. París.

FONSECA, M. \& SANTOS, C., 1993. Relações sociais de gênero, status da mulher e a queda da fecundidade no Brasil: algumas inter-relações. In: La Transición Demográfica en América Latina y el Caribe. IV Conferencia Latinoamericana de Población. Volumen I. Segunda parte, pp. 763-783, Ciudad de México: INEGI (Instituto Nacional de Estadística, Geografía e Informática) - UNAM (Universidad Autónoma de México) - PROLAP (Programa Latinoamericano de Actividades en Población).

FUCARACCIO, A., 1970. El control dela natalidad yel argumento del ahorro y la inversión. México DF: Conferencia Regional Latinamericana de Población.

FUCARACCIO, A., 1994. Temas de Población y Desarrollo. In: Políticas de Población en Centroamérica, El Caribey México. (R. Benítez \& E. Ramírez, org.), pp. 73-101. México: INAP (Instituto Nacional de Administración Pública de Guatemala) - IIS/UNAM (Instituto de Investigaciones Sociales de la Universidad Autónoma de México) PROLAP (Programa Latinoamericano de Actividades en Población).

KLIKSBERG, B., 1993. La Escalada de la Pobreza en América Latina. In: Pobreza. Un Tema Impostergable. Nuevas Respuestas a Nivel Mundial (B. Kliksberg, org.), pp. 7-12. Caracas: FCE (Fondo de Cultura Económica) - CLAD (Centro Latinoamericano de Administración para el Desarrollo) PNUD (Programa de las Naciones Unidas para el Desarrollo).

MCNICOLL, G., 1984. Consequencies of rapid population growth: an overview and assessment. Population and Development Review, 10:177-240.

MASON, O., 1988. The impact of women's position on demographic change during the course of devel- opment: what do we know?. ConferenceWomen's Position and Demographic Change in the Course of Development. America (International Union for the Scientific Study of Population (IUSSP), org.). Oslo: IUSP.

MERTENS, W., 1982. Reflexiones Teórico-Metodológicas sobre Investigaciones en Población. México: El Colegio de México.

NACIONES UNIDAS, 1978. Factores Determinantes y Consecuencias de las Tendencias Demográficas. ST SOA Ser. A50. New York: Naciones Unidas.

NACIONES UNIDAS, 1990. Desarrollo Humano, Informe 1990. New York: Naciones Unidas.

PATARRA, N., 1973. Transición Demográfica: iresumen histórico o teoría de población?. Demografía y Economía, 7:86-95.

PATARRA, N., 1994. Transição demográfica: novas evidências, velhos desafios. Revista Brasileira de Estudos de População, 11:27-40.

PEACOCK, A., 1952. Theory of population and modern economic analysis. Population Studies, 6:114-122.

POPULATION REPORTS, 1994. EI Medio Ambiente y el Crecimiento de la Población: acciones para la presente década. Population Reports, Suplemento Serie M, 10

PRESSAT, R., 1983. El Análisis Demográfico. Métodos, Resultados, Aplicaciones. Ciudad de México: Fondo de Cultura Económica. Segunda Edición en español de la segunda en francés.

RODGERS, G.; HOPKINS, M. \&WERY, R., 1977. Population, Employment and Inequality. BachuePhilipines: OIT (Organización Internacional del Trabajo).

SIM ON, J., 1980. Resources, Population and Environment: an Overssupply of Bad News. Science, 208: 1431-1436.

SINGER, P., 1976. Dinâmica Populacional e Desenvolvimento. O Papel do Crescimento Populacional no Desenvolvimento Econômico. São Paulo: Hucitec.

SINGER, P., 1994. Ética Prática. São Paulo: Martins Fontes.

SZRETER, S., 1993. The idea of demographic transition and study of fertility change. Population and Development Review, 19:659-701.

TAPINOS, G., 1994. Crecimiento Demográfico y Crecimiento Económico. In: Políticas de Población en Centroamérica, El Caribey México. (Benítez, R.; Ramírez, E., eds), pp. 65-72. México: INAP (Instituto Nacional de Administración Pública de Guatemala) - IIS/UNAM (Instituto de Investigaciones Sociales de la Universidad Autónoma de México) - PROLAP (Programa Latinoamericano de Actividades en Población).

WATKINS, S. \& WALLE, E., 1983. Nutrition, mortality and population size: Malthus. Court of Last resort. Journal of Interdisciplinary History, 13:205-226.

ZUMBADO, E., 1993. Prefacio. In: Pobreza, un Tema Impostergable. Nuevas Respuestas a Nivel Mundial (B. Kliksberg, org.), pp. XV-XVII. Caracas: FCE (Fondo de Cultura Económica - CLAD (Centro Latinoamericano de Administración para el Desarrollo) - PNUD (Programa de las Naciones Unidas para el Desarrollo). 